which the refractive index is taken as unity. Thus if points a thousandth of an inch apart, with a total depth of a tenth of an inch, were to be photographed, the value of $K$ would be 1 , and with a perfect lens the definition might be estimated as very good. The attainment of a very good negative would depend on obtaining a large enough image to make the tendeney of photographic images to spread negligible. The sounder practice, though not always possible on account of the space required, appears to be to obtain a picture of the largest size required in a single operation, and not, as has been suggested, the subsequent enlargement of a small original negative.

National Physical Laboratory,

T. SмIтH.

Teddington, Middlesex.

Wiтhout entering into a discussion of the correct interpretation of high power microscopic images, which is a very recondite subject, I should like to point out that Mr. Mallock's statement in NATURE of Oct. 13 that an object of alternate opaque and transparent lines cannot be separated by any method if their spacing is much less than a wave-length, is not confirmed by experience. Lines of this nature on the surface of etched steel have been photographed by visual light, which are not more than $1 / 140,000$ of an inch in their spacing, and can also be seen when approximately of this size. There is some evidence to show that lines nearly twice as fine can be photographed by ultra-violet light of wave-length 1850 .

May I direct Mr. Mallock's attention to a short note on diatom structure in the Journal of the Royal Microscopical Society, vol. 42, pp. 338-339, which indicates another interpretation of the results which he attributes to a lenticular structure in the object observed.

69 Mortimer Street, W.1, Oct. 15.

Influence of Temperature on the Raman Effect.

As has been emphasised by Prof. Pringsheim in his recent admirable report on the Raman effect (Die Naturwissenschaften, Aug. 3, 1928), there is a farreaching and fundamental analogy between the be-

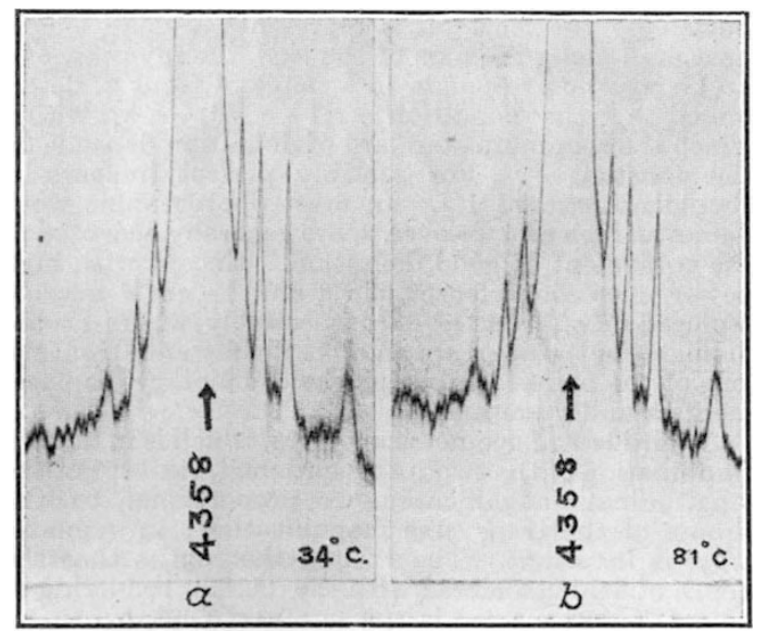

FIG. 1.

haviour of electrons and of light quanta during their collisions with material particles. The new lines appearing in the spectrum of the scattered light are the result of inelastic collisions or of super-elastic collisions of the light quanta with the molecules of the medium, according as the shift of frequency is towards longer or shorter wave-lengths. As has already been pointed out in an earlier communication (NATURE, July 7, 1928, p. 12), the lines with enhanced frequencies are usually of much smaller intensity than those degraded in frequency to an equal extent. The natural explanation of this is that comparatively few molecules are normally present in an excited state and therefore in a position to communicate energy to the light quantum. As the temperature rises, we should expect the number of such molecules to increase, and the proportion of super-elastic to inelastic collisions to rise pari passu.

The case of carbon tetrachloride is very suitable for an experimental test of this point, as there are numerous Raman lines with relatively small shifts of frequency in its scattered spectrum. I have made experiments which confirm this theoretical expectation. Figs. $1 a$ and $1 b$ are microphotometric records of the Raman spectrum of carbon tetrachloride excited by the 4358 group of the mercury arc, the former being taken at $34^{\circ} \mathrm{C}$. and the latter at $81^{\circ} \mathrm{C}$. It will be seen that the lines of higher frequency (towards the left) increase in intensity and those of lower frequency (towards the right) decrease in intensity when the temperature is raised.

210 Bowbazar Street,

Calcutta, Sept. 6.

\section{Elastic Constants of Single-crystal Aluminium Wire.}

IN connexion with my investigations on certain elastic properties of solid metallic elements, Prof. H. C. H. Carpenter, of the Imperial College of Science and Technology, South Kensington, has had made for me single-crystal aluminium wires of diameters $1 \mathrm{~mm}$. and $2 \mathrm{~mm}$. respectively. The wires were drawn down from a single-crystal bar of diameter 0.564 inches and about 4 inches in length.

As a result of a series of carefully conducted experiments, it is found that for these specimens of wires $y=6 \cdot 779 \times 10^{11}$ and $n=2 \cdot 432 \times 10^{11}$ (temp. $28^{\circ}-28 \cdot 5^{\circ} \mathrm{C}$.). The first of these two elastic constants is determined with the help of a sensitive form of extensometer responding to elongations with a few tenths of a gram; and the second, by setting suitable lengths of the wires into torsional vibrations in an air-free chamber. It will be seen that these moduli for single-crystal wires are slightly less than for ordinary specimens.

Vizianagaram, India, Sept. 20.

G. Subrahmaniam.

\section{Geological Jargonese.}

THE correspondent on the above subject in NATURE of Oct. 13 gives expression to a matter of regret for many a lay reader like myself, but that regret should not be sharpened by impatience.

NATURE is a kind of mixed grill, prepared for people of widely different tastes, all of them epicures. Though your lay readers may frequently wish that they could fully understand some of the articles that are written, it should not be forgotten that the real use of NATURE is as a medium of exchange of technical information on subjects embracing the whole field of science, and it is obvious that if the geologist were to choose his language to suit the chemist, or vice versa, the value of his contribution might very well be destroyed.

If voracious feeders will consume mixed dishes, they must not complain if some of the ingredients do not suit their palate. J. P. C. Done.

Cricklewood, N.W.2.

No. 3078 , VoL. 122] 